\title{
Electron Scattering in Hot/Warm Plasmas
}

Balazs F. Rozsnyai

January 25, 2008

Journal of High Energy Density Physics 
This document was prepared as an account of work sponsored by an agency of the United States government. Neither the United States government nor Lawrence Livermore National Security, LLC, nor any of their employees makes any warranty, expressed or implied, or assumes any legal liability or responsibility for the accuracy, completeness, or usefulness of any information, apparatus, product, or process disclosed, or represents that its use would not infringe privately owned rights. Reference herein to any specific commercial product, process, or service by trade name, trademark, manufacturer, or otherwise does not necessarily constitute or imply its endorsement, recommendation, or favoring by the United States government or Lawrence Livermore National Security, LLC. The views and opinions of authors expressed herein do not necessarily state or reflect those of the United States government or Lawrence Livermore National Security, LLC, and shall not be used for advertising or product endorsement purposes. 


\title{
Electron Scattering in Hot/Warm Plasmas. \\ Balazs F. Rozsnyai* \\ Lawrence Livermore National Laboratory \\ P.O. Box 808 \\ Livermore, California 94550.
}

Key words: Electrical and thermal conductivity, electron scattering.

PACS: $52.25 \mathrm{Fi}, 52.27 \mathrm{Gr}, 34.80 .-\mathrm{i}$

\begin{abstract}
.
Electrical and thermal conductivities are presented for aluminum, iron and copper plasmas at various temperatures, and for gold between 15000 and 30000 Kelvin. The calculations are based on the continuum wave functions computed in the potential of the temperature and density dependent self-consistent 'average atom' (AA) model of the plasma. The cross sections are calculated by using the phase shifts of the continuum electron wave functions and also in the Born approximation. We show the combined effect of the thermal and radiative transport on the effective Rosseland mean opacities at temperatures from 1 to $1000 \mathrm{eV}$. Comparisons with low temperature experimental data are also presented.
\end{abstract}

\section{Introduction.}

The objective of this paper is to show the effects of the details of electron scattering cross sections on the transport properties of plasmas. The key element is the electron momentum transfer cross section that enters into the formulas for both the electrical and thermal conductivities. The potential in which the electrons scatter is a basic element and that is taken as an input from the author's temperature and density dependent self-consistent model for the plasma [1], that was also used for the computation of radiative properties $[2,3,4]$. For the computation of the electrical resistivity we use the Ziman formula $[5,6,7]$, whereas for the thermal conductivity we adopt the method of L. Mestel $[8,9]$. No restrictions are made for the degree of degeneracy of the electron gas, so our model is applicable for any temperature and density. However, the continuum wave functions of the scattering states are computed by the non-relativistic Schrodinger equation, even if the self-consistent potential is based on the DiracSlater model. The ratio of the small/large components of the Dirac equation is of the order of $\mathbf{c p} /\left(\mathbf{E}+\mathbf{m c}^{2}\right)$ and for a $1 \mathrm{KeV}$ free electron the above ratio is about $4 \%$. Actually, the fact that the wave functions have to be properly normalized improves the above error for cross section calculations, but the author has no detailed estimates for that. In the next section we present the basics of the theory and in Section III we present computed data together with experimental comparisons.

*Corresponding author: e-mail: rozsnyai1@1lnl.gov, ROZSNYAI9@aol.com 


\section{Theory.}

We compute the electrical resistivity given by Ziman's formula $[5,6]$

$$
\mathbf{R}=-\frac{\hbar}{3 \pi\left(\mathbf{Z}^{*}\right)^{2} \mathbf{e}^{2} \boldsymbol{\rho}_{\mathrm{i}}} \int_{0}^{\infty} \mathbf{f}^{\prime}(\varepsilon) \mathbf{d} \varepsilon \int_{0}^{2 \mathbf{k}} \boldsymbol{\sigma}(\mathbf{K}) \mathbf{K}^{3} \mathbf{d K S}(\mathbf{K})(\mathrm{sec})
$$

In $\operatorname{Eq}(1) \rho_{i}$ is the ion density, $Z^{*}$ the effective charge of the central ion, $\varepsilon$ the energy of the incident electron, $\mathbf{f}^{\prime}(\boldsymbol{\varepsilon})$ the derivative of the Fermi function and $\sigma(\mathrm{K})$ is the differential scattering cross section in terms of the momentum change variable $\mathrm{K}$. The later is related to the momentum vector of the incident electron by $\mathbf{K}^{2}=2 \mathbf{k}^{2}[1-\cos (\boldsymbol{\vartheta})]$ where $\boldsymbol{\vartheta}$ is the scattering angle and the energy of the incident electron is $\varepsilon=\frac{\hbar^{2} \mathbf{k}^{2}}{2 \mathbf{m}}$. The quantity $\mathrm{S}(\mathrm{k})$ in the second integral is the structure factor to account for multiple scattering by many ions in the plasma, and it is related to the Fourier transform of the ion distribution by

$$
\mathbf{S}(\mathbf{K})=1+4 \pi \rho_{\mathbf{i}} \int_{0}^{\infty}\left[\mathbf{g}_{\mathbf{i}}(\mathbf{r})-1\right]^{\sin \mathbf{K r}} \mathbf{K r} \mathbf{r}^{2} \mathbf{d r}
$$

where $\mathbf{g}_{\mathbf{i}}(\mathbf{r})$ is the ion distribution. In this work we use the ion-sphere model that defines the ion-

sphere radius by $\mathbf{r}_{0}^{3}=\frac{3}{4 \pi \rho_{\mathrm{i}}}$, and for the ion distribution function we take a simple step function $\mathbf{g}_{\mathbf{i}}(\mathbf{r})=0$ if $\mathbf{r}<\mathrm{r}_{0}$

$$
=1 \text { if } \mathbf{r}>\mathrm{r}_{0} \quad \text { and obtain } \quad \mathbf{S}_{\text {is }}(\mathbf{K})=1-\frac{3}{\left(\mathbf{K r}_{0}\right)^{3}}\left[\sin \mathbf{K} \mathbf{r}_{0}-\mathbf{K} \mathbf{r}_{0} \cos \mathbf{K} \mathbf{r}_{0}\right] \text {, }
$$

the ion-sphere structure factor, which was also used in Ref.[5].

In Equation(1) the second integral is related to the general formula of momentum transfer cross section by

$$
\sigma^{\mathrm{M}}(\varepsilon)=\frac{\pi}{\mathrm{k}^{4}} \int_{0}^{2 \mathrm{k}} \sigma(\mathrm{K}) \mathrm{S}(\mathrm{K}) \mathrm{K}^{3} \mathrm{dK}
$$

Using Eq.(4) we have for the resistivity

$$
\mathbf{R}=\frac{4}{3} \frac{1}{\pi^{2}} \frac{1}{\left(\mathbf{Z}^{*}\right)^{2}} \frac{\mathbf{m}^{2}}{\hbar^{3}} \frac{1}{\mathbf{e}^{2} \boldsymbol{\rho}_{\mathbf{i}}} \int_{0}^{\infty} \mathbf{f}^{\prime}(\boldsymbol{\varepsilon}) \varepsilon^{2} \boldsymbol{\sigma}^{\mathbf{M}}(\boldsymbol{\varepsilon}) \mathbf{d} \boldsymbol{\varepsilon} \quad(\mathrm{sec})
$$

In the Born approximation the computation of $\boldsymbol{\sigma}(\mathbf{K})$ involves the Fourier transform of the scattering potential, since the scattering amplitude is

$$
\mathbf{f}(\vartheta \varphi)=-\frac{1}{2 \pi} \frac{\mathbf{m}}{\hbar^{2}} \int \mathrm{e}^{\mathrm{i} \mathbf{K} \cdot \vec{r}} \mathbf{V}(\overrightarrow{\mathbf{r}}) \mathbf{d}^{3} \mathbf{r}=-4 \pi^{2} \frac{\mathbf{m}}{\hbar^{2}} \mathbf{V}(\mathbf{K}) .
$$

However, as we will see later, the Born approximation frequently overestimates the cross sections and one needs more accurate quantum mechanical treatment. In the case when the scattering is by a single center, when $\mathrm{S}(\mathrm{K})=1$, the momentum transfer cross section is given by

$$
\mathbf{\sigma}_{\mathrm{s}}^{\mathrm{M}}=\frac{4 \pi}{\mathbf{k}^{2}} \sum_{\mathbf{I}}(\mathbf{l}+1) \sin ^{2}\left(\boldsymbol{\delta}_{\mathbf{I}}-\boldsymbol{\delta}_{\mathbf{I + 1}}\right)
$$

where $\delta_{1}$ is the phase shift of the continuum state with angular momentum quantum number 1 . 
In the presence of the structure factor things are a bit more complicated and in terms of the phase shifts and Legendre polynomials we obtain

$$
\begin{aligned}
& \boldsymbol{\sigma}^{\mathbf{M}}=\frac{\pi}{\mathbf{k}^{6}} \sum_{\mathrm{I}} \sum_{\mathbf{I}^{\prime}}(2 \mathbf{I}+1)\left(2 \mathbf{I}^{\prime}+1\right) \mathbf{e}^{\mathbf{i}\left(\boldsymbol{\delta}_{\mathbf{l}}-\boldsymbol{\delta}_{\mathbf{I}^{\prime}}\right)} \sin \boldsymbol{\delta}_{\mathbf{I}} \sin \boldsymbol{\delta}_{\mathbf{I}^{\prime}} \mathbf{I}_{\mathbf{I I}^{\prime}}= \\
& \frac{\pi}{\mathbf{k}^{6}}\left\{\sum_{\mathbf{I}}(2 \mathbf{l}+1)^{2} \sin ^{2} \boldsymbol{\delta}_{\mathbf{I}} \mathbf{I}_{\mathbf{I I}}(\mathbf{k})+\sum_{\mathbf{I}} \sum_{\mathbf{I}^{\prime}>\mathbf{1}}(2 \mathbf{l}+1)\left(2 \mathbf{l}^{\prime}+1\right) 2 \cos \left(\boldsymbol{\delta}_{\mathbf{I}}-\boldsymbol{\delta}_{\mathbf{I}^{\prime}}\right) \sin \boldsymbol{\delta}_{\mathbf{I}} \sin \boldsymbol{\delta}_{\mathbf{I}^{\prime}} \mathbf{I}_{\mathbf{I I}}(\mathbf{k})\right\} \\
& \text { with } \\
& \mathbf{I}_{I^{\prime}}=\int_{0}^{2 \mathbf{k}} \mathbf{P}_{\mathbf{l}}\left(1-\frac{\mathbf{K}^{2}}{2 \mathbf{k}^{2}}\right) \mathbf{P}_{\mathbf{I}^{\prime}}\left(1-\frac{\mathbf{K}^{2}}{2 \mathbf{k}^{2}}\right) \mathbf{K}^{3} \mathbf{S}(\mathbf{K}) \mathbf{d K}
\end{aligned}
$$

It should be noted that when $\mathrm{S}(\mathrm{K}) \equiv 1$ by virtue of the properties of the Legendre polynomials

$$
\int_{0}^{2 \mathbf{k}} \mathbf{P}_{\mathbf{l}}^{2}\left(1-\frac{\mathbf{K}^{2}}{2 \mathbf{k}^{2}}\right) \mathbf{K}^{3} \mathbf{d K}=\frac{4 \mathbf{k}^{4}}{2 \mathbf{l}+1} \text { and } \int_{0}^{2 \mathbf{k}} \mathbf{P}_{\mathbf{l}}\left(1-\frac{\mathbf{K}^{2}}{2 \mathbf{k}^{2}}\right) \mathbf{P}_{\mathbf{l}+1}\left(1-\frac{\mathbf{K}^{2}}{2 \mathbf{k}^{2}}\right) \mathbf{K}^{3} \mathbf{d} \mathbf{K}=\frac{4 \mathbf{k}^{4}(\mathbf{l}+1)}{(2 \mathbf{l}+1)(2 \mathbf{l}+3)}
$$

Equation(8) gives back Equation(7).

For the computation of thermal conductivity we adopt the method of Mestel $[8,9]$ and use

$$
\lambda_{\mathbf{c}}=\frac{16 \mathbf{m}(\mathbf{k T})^{4}}{3 \mathbf{h}^{3} \mathbf{e}^{4} \mathbf{Z}^{* 2} \rho_{\mathbf{i}}} \mathbf{L}(\boldsymbol{\eta}) \quad\left(\mathrm{cm}^{-1} \sec ^{-1}\right)
$$

where the integral $L$ depends on the electron degeneracy parameter $\eta$ and on the momentum transfer cross section by

$$
\mathbf{L}(\eta)=\frac{\left(\int_{0}^{\infty} \frac{\varepsilon^{4}}{\theta(\varepsilon)} \mathbf{f}^{\prime}(\varepsilon) \mathbf{d} \varepsilon\right)^{2}}{\int_{0}^{\infty} \frac{\varepsilon^{3}}{\theta(\varepsilon)} \mathbf{f}^{\prime}(\varepsilon) d \varepsilon}-\int_{0}^{\infty} \frac{\varepsilon^{5}}{\theta(\varepsilon)} \mathbf{f}^{\prime}(\varepsilon) \mathbf{d} \varepsilon
$$

Where $f^{\prime}$ is again the derivative of the Fermi function and the function $\theta$ is related to the momentum transfer cross section by

$$
\boldsymbol{\theta}(\boldsymbol{\varepsilon})=\frac{\hbar^{4}}{\mathbf{m}^{2} \mathbf{e}^{4}} \frac{1}{\mathbf{Z}^{* 2}} \int_{0}^{2 \mathbf{k}} \boldsymbol{\sigma}(\mathbf{K}) \mathbf{K}^{3} \mathbf{d} \mathbf{K}=\frac{\hbar^{4}}{\mathbf{m}^{2} \mathbf{e}^{4}} \frac{1}{\mathbf{Z}^{* 2}} \frac{\mathbf{k}^{4}}{\pi} \boldsymbol{\sigma}^{\mathbf{M}}(\mathbf{k}) \quad \text { (dimensionless). }
$$

In Refs $[8,9]$ and in many other textbooks $\theta$ is calculated by assuming Coulomb scattering which yields

$$
\boldsymbol{\theta}_{\mathrm{c}}=\frac{1}{2} \ln \frac{2}{1-\cos \boldsymbol{\vartheta}_{0}} ; \boldsymbol{\vartheta}_{0}=\frac{\hbar}{\mathbf{m v R}_{\mathrm{z}}}
$$

where $R_{Z}$ is the impact parameter. Also in Refs. [8,9] the function $\theta(\varepsilon)$ is taken with an average value $\varepsilon_{\text {av }}$ outside the integrals in (9a) which yields

$$
\mathbf{L}(\boldsymbol{\eta})=\frac{1}{\boldsymbol{\theta}\left(\boldsymbol{\varepsilon}_{\mathrm{av}}\right)} \frac{15 \mathbf{F}_{2}(\boldsymbol{\eta}) \mathbf{F}_{4}(\boldsymbol{\eta})-16 \mathbf{F}_{3}^{2}(\boldsymbol{\eta})}{3 \mathbf{F}_{2}(\boldsymbol{\eta})}
$$

where now the F-s are Fermi-Dirac integrals of integer indices. In the author's experience both the Coulomb scattering assumption and taking an average value for $\theta(\varepsilon)$ have limited validity. 
For the calculation of phase shifts of the $l$ spherical waves we use two different methods; the first is based on the Sommerfeld -Bohr (SB) quantization rule

$$
\delta_{\mathbf{l}}^{\mathbf{S B}}(\boldsymbol{\varepsilon})=\int_{\mathbf{r}_{\mathbf{i}}}^{\mathbf{r}_{0}}\left\{\left[2 \boldsymbol{\varepsilon}-2 \mathbf{V}(\mathbf{r})-\frac{(\mathbf{l}+1 / 2)^{2}}{\mathbf{r}^{2}}\right]^{1 / 2}-\left[2 \boldsymbol{\varepsilon}-\frac{(\mathbf{l}+1 / 2)^{2}}{\mathbf{r}^{2}}\right]^{1 / 2}\right\} \mathbf{d r}
$$

where $\mathbf{V}(\mathbf{r})$ is the scattering potential , $\mathbf{r}_{\mathbf{i}}$ is the inner turning point radius (where the integrand becomes imaginary) and $\mathbf{r}_{0}$ is the ion-sphere radius beyond which the potential can be neglected. The SB quantization rule requires only the potential. Our second method employs the computation of the exact quantum -mechanical phase shift $\delta_{1}$ that uses both the continuum wave function $\mathbf{R}_{\mathbf{l}}(\mathbf{r})$ and the potential $\mathbf{V}(\mathbf{r})$, and it is given by

$$
\operatorname{tn} \delta_{1}(k)=\frac{k \mathbf{j}_{1}{ }^{\prime}\left(k \mathbf{r}_{0}\right)-\gamma \mathbf{j}_{\mathbf{1}}\left(\mathbf{k r}_{0}\right)}{\mathbf{k n}_{1}^{\prime}\left(\mathbf{k r}_{0}\right)-\gamma \mathbf{n}_{\mathbf{l}}\left(\mathbf{k r}_{0}\right)}
$$

where $j_{1}$ and $n_{1}$ stand for the spherical Bessel and Neumann functions, respectively and $\gamma$ is the logarithmic derivative of the radial function $\mathbf{R}_{\mathbf{l}}\left(\mathbf{r}_{0}\right)$ at the ion-sphere radius. With regard to Equations (11a) and (11b) it is instructive to make a few remarks about Levinson's theorem regarding the zero energy phase shifts

$$
\delta_{1}(0)=n_{1} \pi
$$

where $n_{1}$ is the number of bound states with angular momentum quantum number 1 in the potential. Within the (SB) quantization rule Equation (11a) accounts for Levinson's theorem but Eq(11b) does not. Therefore, when the phases shifts themselves are computed from $\mathrm{Eq}(11 \mathrm{~b})$ the zero energy phase shifts have to be added to the arctangent of $\mathrm{Eq}(11 \mathrm{~b})$ and then the phase shifts vary from that value in a continuous fashion..

Finally, to compare the electron and photon transport processes we need the electron conductive opacity defined by

$$
\boldsymbol{\kappa}_{\mathbf{c}}=\frac{4 \mathbf{a c}(\mathbf{k T})^{3}}{3 \rho \lambda_{\mathbf{c}}}\left(\mathrm{cm}^{2} / \mathrm{g}\right)
$$

where $\rho$ is the matter density in $\mathrm{g} / \mathrm{cm}^{3}$ and $\mathbf{a}=\frac{\pi^{2}}{15} \frac{1}{(\hbar \mathbf{c})^{3}}$, the Stephan-Boltzmann constant in units of (ev.cm $)^{-3}$. The electron conductive opacity and the radiative Rosseland mean opacity add together reciprocally like parallel resistors giving an effective Rosseland mean opacity

$$
\frac{1}{\mathbf{K}_{\text {eff }}}=\frac{1}{\mathbf{K}_{\text {rad }}}+\frac{1}{\mathbf{K}_{\mathbf{c}}}
$$

The radiative part in a Planckian radiation field is given by

$$
\frac{1}{\mathbf{K}_{\mathrm{rad}}}=\frac{15}{4 \pi^{4}} \frac{1}{(\mathbf{k T})^{5}} \int_{0}^{\infty} \frac{(\mathbf{h} \boldsymbol{v})^{4} \exp (\mathbf{h} \mathbf{v} / \mathbf{k T})}{[\exp (\mathbf{h} \mathbf{v} / \mathbf{k T})-1]^{2}} \frac{1}{\boldsymbol{\sigma}(\boldsymbol{v})} \mathbf{d}(\mathbf{h} \mathbf{v})
$$

where $\boldsymbol{\sigma}(\boldsymbol{v})$ is the frequency dependent photoabsorption cross section. 


\section{Computations.}

In this Section we present computed data for aluminum, iron and copper. Actually, we present electrical conductivity, which is the reciprocal of the resistivity, together with thermal conductivity. The aim of this paper is twofold; to present high temperature calculations where radiation energy transport is important and to see the extent electron transport competes with radiation transport and to present low temperature calculations where experimental data are available. As was stated before, the computations use the self-consistent potentials and other EOS data as inputs from a self-consistent Dirac-Slater model that was published before $[1,2,3,4]$, the main part of this paper is the computation of scattering states, phase shifts and structure factors. The author does not intend to present an unnecessary set of redundant figures, so we illustrate the physics details for aluminum only, which are quite similar for the other two elements. For iron and copper we present only the essential results. We summarize some of the plasma conditions in tables.

Figure 1 shows the self-consistent potentials for aluminum at metallic density, $2.7 \mathrm{~g} / \mathrm{cc}$ at four temperatures. The potential, V(r) is given by a self-consistent Dirac-Slater model [1], and contains all the interactions. The exchange-correlation part is temperature-density dependent [10] and it behaves as $\sim 1 / \mathrm{kT}$ as the temperature increases. We show $-\mathrm{rV}(\mathrm{r})$, which has to be $\mathrm{Z}$ at the nucleus.

We show some detailed physics examples on Figures 2 and 3. In Figures $2 \mathrm{a}$ and $2 \mathrm{~b}$ show the phase shifts both for the SB quantization rule and for the quantum-mechanical rule as given by Equations (11a) and (11b), respectively. In general, the differences between the two ways of calculating the phase shifts are minor. Exceptions may occur at low incident electron energy by virtue of Levinson's theorem. If there is a nearly bound resonant state with angular momentum quantum number 1, then the continuum wave function changes rapidly at low energy and the phase shifts given by Eqs.(11a) and (11b) may differ appreciably. This is evident for the $1=2$ phase shifts shown in Fig. 2 a and the difference becomes small again for $1=3$. Actually, the rapid rise of the quantum mechanical phase shift with $1=2$ is associated with a resonant $\mathrm{d}$ state around 8 $\mathrm{eV}$. It is also evident that as the quantum number $l$ increases the differences between the $\delta_{l}$ and $\delta_{l+l}$ phase shifts rapidly disappear which assures the convergence of the infinite series in Equations (7) and (8) for the momentum transfer cross sections. In Fig. $2 b$ for $1>9$ the SB and quantum mechanical phases shifts seem to diverge above $5000 \mathrm{eV}$. That also happens at higher 1 states at high incident electron energy. However, the important feature, namely the diminishing difference between the $\delta_{l}$ and $\delta_{l+1}$ phase shifts remains for both cases. Actually, in this work we gave allowances to go as high as 30 for the $l$ quantum number. In Figures $3 \mathrm{a}$ and $3 \mathrm{~b}$ we show the momentum transfer cross sections of aluminum based on various assumptions at normal density, $2.7 \mathrm{~g} / \mathrm{cc}$, and in Figures $4 \mathrm{a}$ and $4 \mathrm{~b}$ we show the electrical and thermal conductivities. The room temperature data marked by red dots were taken form the Handbook of Physics and

Chemistry[11]

The main effect of the structure factor is that it depresses the cross sections at low energies as given by Eq.(4) and as is apparent in the figures showing the momentum transfer cross sections. In Figure 5 we compare the structure factor based on the ion-sphere model with that of the more sophisticated molecular dynamics calculations of Young Corey and DeWitt (YCD) [12] for two typical cases. It appears that the steep ascending regions of the YCD and ion-sphere structure factors are close. 
In Figure 6 we show the effective Rosseland mean opacities for aluminum as given by Eq.(13) under various assumptions. To illustrate the significance of the electron scattering we also show the radiative part alone. The results obtained by the simple Coulomb scattering, as given by Eq.(10c), are also shown. The structure factors make a minimal difference in the total Rosseland mean opacities, and we illustrate the difference only for the usage of the SB phase shifts. Figures $7 \mathrm{a}$ and $7 \mathrm{~b}$ show the same for iron as Figures $4 \mathrm{a}$ and $4 \mathrm{~b}$ for aluminum and Fig. 8 shows the Rosseland mean values for iron. In Figures 9 and 10 we show the conductivities and Rosseland mean opacities for copper at metallic density. Comparing Figures 6, 8 and 10 we notice that the radiative part of the Rosseland mean of copper shows a maximum around $8 \mathrm{eV}$ temperature. This is a typical feature of the band structure predicted by the ion-sphere model. For aluminum at $1 \mathrm{eV}$ temperature the overlapping $3 \mathrm{p}$ and $3 \mathrm{~s}$ bands are populated by only three electrons thus allowing intraband transitions which behave like free-free transitions. Similar is the case for iron with 8 electrons in the overlapping $3 \mathrm{~d}$ and $4 \mathrm{~s}$ bands. On the other hand for copper the $3 \mathrm{~d}$ band is completely filled that causes a depressed free-free opacity at $1 \mathrm{eV}$. It is apparent from Figures 6, 8 and 10 that the assumption of Coulomb scattering overestimates the Rosseland mean values even more than the Born approximation using the self-consistent potentials. This point is frequently ignored in many research papers and textbooks and it is the author's intention to emphasis this.

In Tables I.-III we summarize the basic EOS data for the conditions of Figures 1-10.

The last column under $\mathrm{Cl}$ contains the value of continuum lowering at the ion-sphere radius $\mathrm{r}_{0}$. We note that the value of the continuum lowering is exclusively due to the electron exchange, which is temperature dependent in the form $\sim 1 / \mathrm{kT}[10]$, thus reflecting the decreasing value of the continuum lowering with increasing temperature. The value of the plasma coupling parameter $\Gamma$ is not necessarily a monotonic decreasing function of the temperature due to increasing ionization, as is the case for iron.

Table I. ${ }^{\#}$ Temperature, effective charge, Fermi level, plasma coupling parameter and continuum lowering for aluminum at $2.7 \mathrm{~g} / \mathrm{cc}$ density.

$\begin{array}{ccccc}\mathrm{kT}(\mathrm{eV}) & \mathrm{Z}^{*} & \mu(\mathrm{eV}) & \Gamma & \mathrm{Cl}(\mathrm{eV}) \\ & & & & \\ 0.0258 & 2.92 & 11.04 & 1748.2 & -14.7 \\ 1.00 & 2.92 & 10.96 & 45.10 & -14.7 \\ 5.00 & 2.95 & 8.48 & 9.11 & -8.86 \\ 10.00 & 2.98 & 2.197 . & 4.67 & -6.50 \\ 50.00 & 5.37 & -93.11 & 3.08 & -3.26 \\ 100.00 & 7.33 & -259.8 & 2.88 & -2.32 \\ 500.00 & 11.83 & -2272.6 & 1.50 & -0.777 \\ 1000.00 & 12.81 & -5506.6 & 0.88 & -0.425\end{array}$

\# The Fermi level and number of free electrons are computed from the continuum lowering. 
Table II. The same as Table1 for iron at $7.86 \mathrm{~g} / \mathrm{cc}$ density

$\begin{array}{ccccc}\mathrm{kT}(\mathrm{eV}) & \mathrm{Z}^{*} & \mu(\mathrm{eV}) & \Gamma & \mathrm{Cl}(\mathrm{eV}) \\ 0.0258 & 1.625 & 16.19 & 613.7 & -16.38 \\ 1.00 & 1.900 & 15.36 & 21.44 & -16.43 \\ 5.00 & 2.20 & 0.679 & 5.826 & -11.99 \\ 10.00 & 2.889 & 0.465 & 5.004 & -8.99 \\ 50.00 & 7.181 & -54.20 & 6.182 & -5.95 \\ 100.00 & 10.385 & -191.24 & 6.467 & -4.47 \\ 500.00 & 19.980 & -1838.09 & 4.774 & -1.78 \\ 1000.00 & 22.91 & -4584.69 & 3.135 & -1.03\end{array}$

Table III. Same as table I for copper at $8.96 \mathrm{~g} / \mathrm{cc}$ density.

$\begin{array}{lrrcr}\mathrm{kT}(\mathrm{eV}) & \mathrm{Z}^{*} & \mu(\mathrm{eV}) & \Gamma & \mathrm{Cl}(\mathrm{eV}) \\ 0.0258 & 1.06 & 9.20 & 260.81 & -14.84 \\ 1.00 & 1.08 & 9.19 & 6.998 & -14.89 \\ 5.00 & 4.08 & 9.01 & 20.49 & -13.12 \\ 10.00 & 4.14 & 8.98 & 9.968 & -12.65 \\ 50.00 & 7.96 & -52.72 & 7.605 & -6.14 \\ 100.00 & 11.19 & -180.73 & 7.506 & -4.61 \\ 500.00 & 21.37 & -1803.44 & 5.478 & -1.89 \\ 1000.00 & 25.13 & -4489.21 & 3.790 & -1.12\end{array}$

Experimental data that can serve for the purpose of comparison are available mainly in the low temperature (10000-30000 Kelvin) region. Measured data of electrical conductivities in aluminum and copper plasmas were reported by DeSilva and Katsouros [13], of iron and of other metal plasmas by DeSilva and Rakhel [14].

We show calculated electrical and thermal conductivities as a function of density at $10000 \mathrm{~K}$ $(0.8617 \mathrm{eV})$ temperature on Figures 11,12 and 13 for aluminum, iron and copper. For aluminum and copper we also show the electrical conductivities calculated by the Desjarlais-Lee-More model (DLM) [15]. As is evident form the figures, the results of the phase shift calculations (quantum mechanical or SB) are reasonably close to the experiments, whereas the Born approximation is not very good.

High $\mathrm{Z}$ elements are also of interest, so we show some calculations for gold at $0.5 \mathrm{~g} / \mathrm{cc}$ density, which was also investigated by Renaudin et al. [16]. What is interesting in this case is to see that the Born approximation is totally inadequate.

\section{Discussion.}


We have presented calculations for electrical and thermal conductivities due to electron scattering on the basis of the elements of scattering theory. The Born approximation overestimates the momentum transfer cross sections and it approaches those calculated by the exact or SB phases shifts asymptotically at high electron energies. This is a well known property of the Born approximation and it should be used only when the continuum electrons have much higher energy than the scattering potential, which is seldom the case even for medium Z elements. The assumption of Coulomb scattering seems to be inadequate, especially for dense plasmas, because of the screening by both bound and free electrons. The phase shifts computed by exact quantum mechanics or by the Sommerfeld-Bohr quantization rule predict results that show noticeable differences at low temperature and they seem to converge to each other with increasing temperature. The same conclusion is valid with respect to the inclusion of the structure factor in the momentum transfer cross section. For thermal conductivities the author has only room temperature experimental data as given by Ref.[11]. With regard to those we can observe that the usage of the quantum mechanical phases shifts combined with the structure factor comes closest to the experimental data at room temperature. It should be noted that the computations presented in this paper are based on the ion-sphere model, which is different from the so called 'muffin- thin' model [17] used in the Purgatorio code [18]. In the muffin thin model the electron charge is normalized within the ion sphere radius, but the boundary conditions for the bound states are given at infinity, thus the muffin thin model cannot account for bands. In the ion sphere model the boundary conditions are given at the ion-sphere radius (see Ref.[1]) and the model predicts the transition from a bona fide bound state to bands in a continuous fashion with increasing density. This difference predicts different self-consistent electron potentials which may be important at low temperatures. The author is not aware of accurate experimental data which could clearly favor one model versus the other, so at present the two models should be looked upon comparatively.

\section{Acknowledgements.}

The author is grateful to Dr. A. W. DeSilva of the University of Maryland for supplying the experimental data for aluminum, iron and copper. Thanks are due to Dr. John I. Castor of the Lawrence Livermore National Laboratory for performing the calculations based on the Desjarlais-Lee-More model.

This work performed under the auspices of the U. S. DOE by LLNL under Contract DE-AC52-07NA27344.

\section{References.}

[1] B. F. Rozsnyai, Phys. Rev. A5, 1137 (1972).

[2] A. Goldberg, B. F. Rozsnyai and P. Thompson, Phys. Rev. A34, 421 (1986).

[3] B. F. Rozsnyai, Phys. Rev. A43, 3035 (1991)

[4] B. F. Rozsnyai, S. D. Bloom and D. A. Resler, Phys. Rev. A44, 6791 (1991)

[5] J. M. Ziman, Philos. Mag. 6, 1013 (1961)

[6] F. Perrot and M. W. C. Dharma-wardana, Phys. Rev. A36, 238 (1987) 
[7] See also J.-P. Hansen and I. R. McDonald 'Theory of Simple Liquids' Academic Press (1986)

[8] L. Mestel, Proc. Cambridge Phil. Soc. 46, 331 (1950)

[9] See also Donald D. Clayton, Principles of Stellar Evolution And Nucleosynthesis' McGraw -Hill (1968)

[10] U. Gupta and A. K. Rajagopal, Phys. Rev. A21, 2064 (1980).

[11] Handbook of Physics and Chemistry. 1970-71, 51-st edition. The Chemical Rubber Co.

[12] D.A. Young, E. M. Corey and H. E. DeWitt. Phys. Rev. A44, 6508, (1991)

[13] A. W. DeSilva and J. D. Katsouros. Phys. Rev. E57, 5945 (1998)

[14] A. W. DeSilva and A. D. Rakhel, Contrib. Plasma Phys. 45,236, (2005)

[15] M. P. Desjarlais, Contrib. Plasma Phys. 41, 267 (2001)

[16] P. Renaudin, V. Recoules, P.Noiret and J. Clerouin, Phys. Rev. E73, 056403 (2006)

[17] D.A. Liberman, Phys. Rev. B20, 4981 (1979)

[18] P.A. Sterne, S.B. Hansen, B.G. Wilson and W.A. Isaacs, HEDP 3, 278 (2007)

Figures.

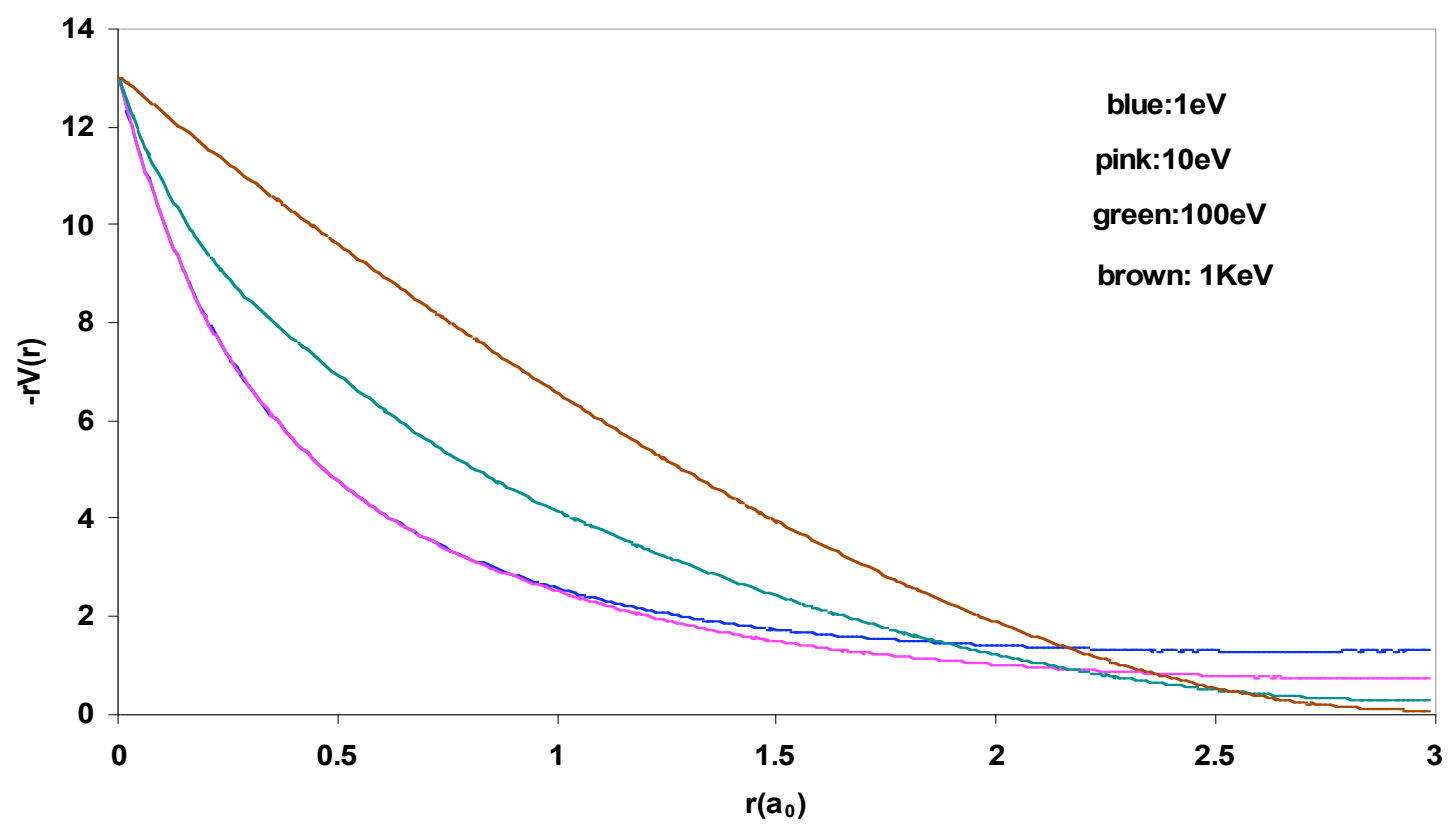

Fig. 1. Self-consistent potentials $-\mathrm{rV}(\mathrm{r})$ of aluminum at $2.7 \mathrm{~g} / \mathrm{cc}$ density. 

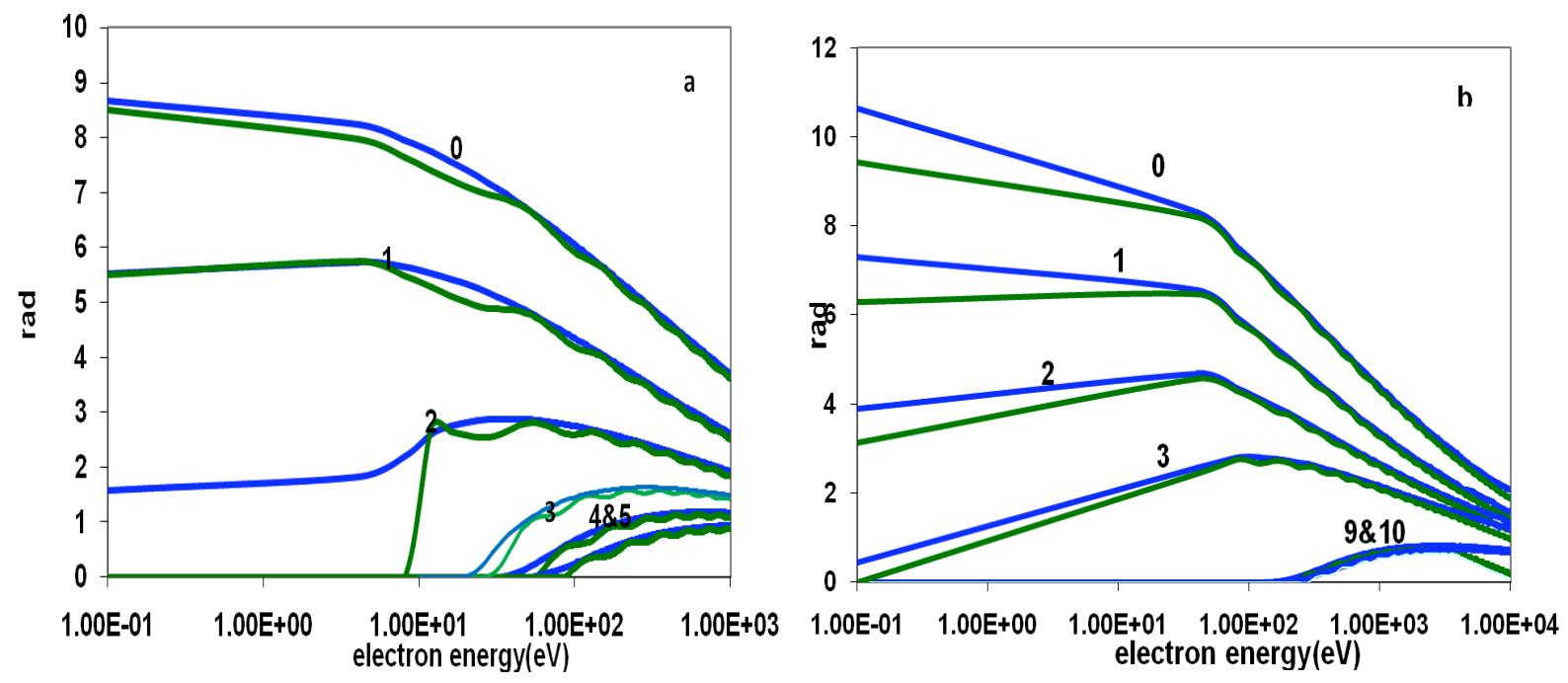

Figure 2. Phase shifts in aluminum at $2.7 \mathrm{~g} / \mathrm{cc}$ density, a, $\mathrm{kT}=100 \mathrm{eV}, \mathrm{b}, \mathrm{kT}=1 \mathrm{KeV}$. The blue and green curves with the Sommerfeld-Bohr and quantum mechanical phase shifts, respectively. The $l$ angular momentum quantum numbers are indicated at the curves.
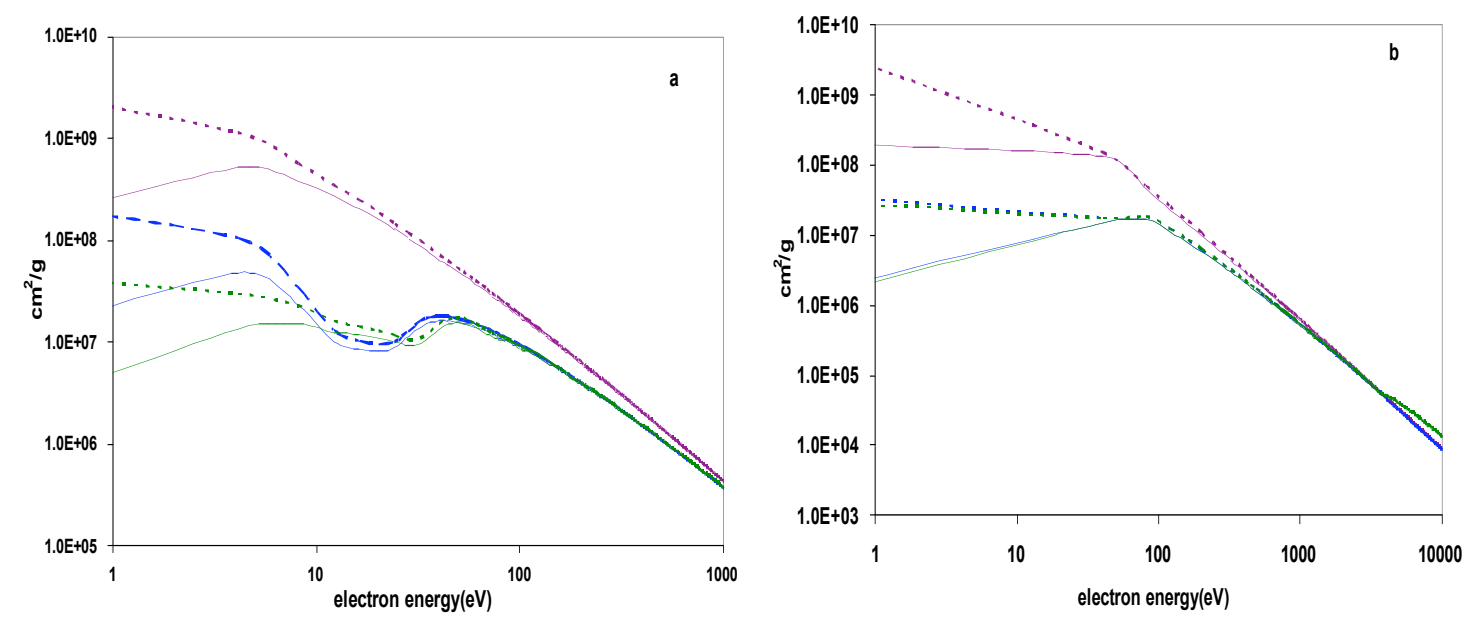

Fig. 3. Momentum transfer cross sections in units of $\mathrm{cm}^{2} / \mathrm{g}$ of aluminum at $2.7 \mathrm{~g} / \mathrm{cc}$ density, a: $k T=100 \mathrm{eV}, \mathrm{b}: \mathrm{kT}=1 \mathrm{KeV}$. The blue and green curves indicate the results of the Sommerfeld-Bohr and quantum mechanical phase shifts, respectively. The violet curves are the results of the Born approximation. The dashed curves indicate $S(K)=1$ for the structure factor. 

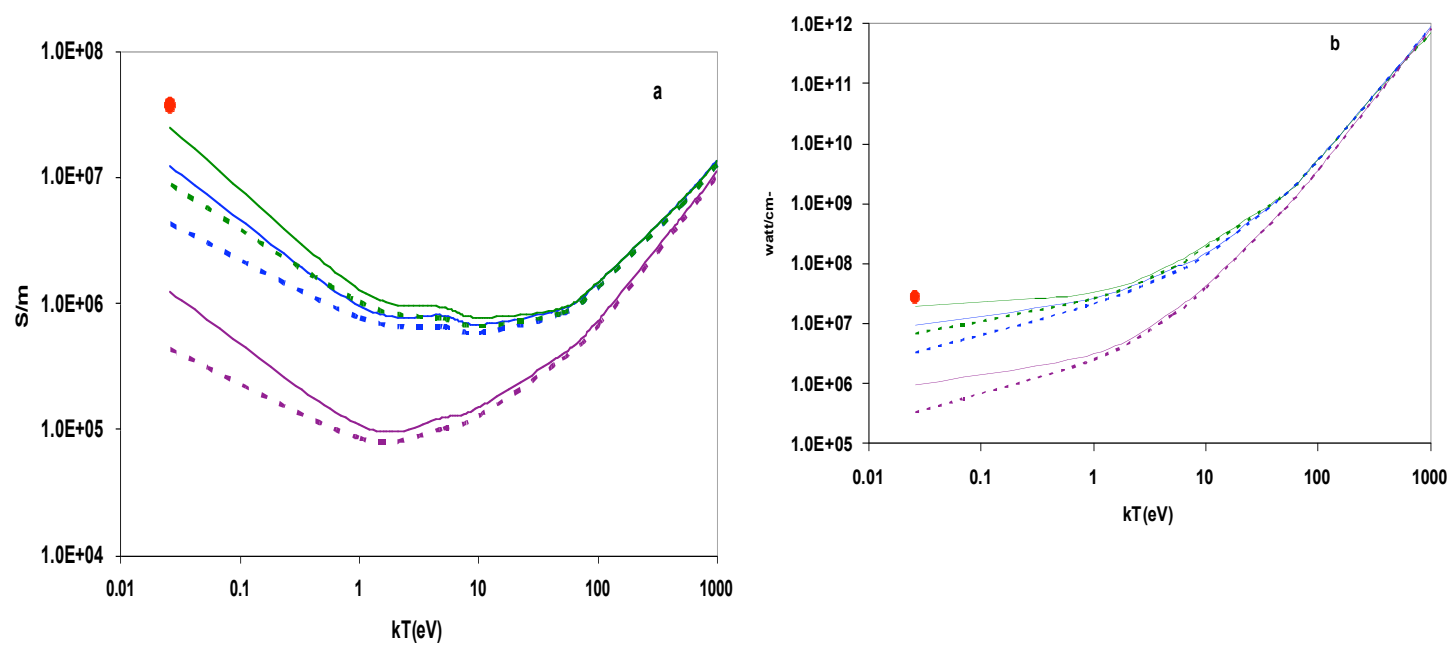

Figure 4. DC electrical conductivities in units of $\mathrm{S} / \mathrm{m}$. (a), and thermal conductivities in units of watt $/ \mathrm{cm}-\mathrm{KeV}$, (b) versus temperature in aluminum at $2.7 \mathrm{~g} / \mathrm{cc}$ density. The colors and dashed lines have the same meanings as in Fig.3. The red dots indicate the experimental room temperature data.

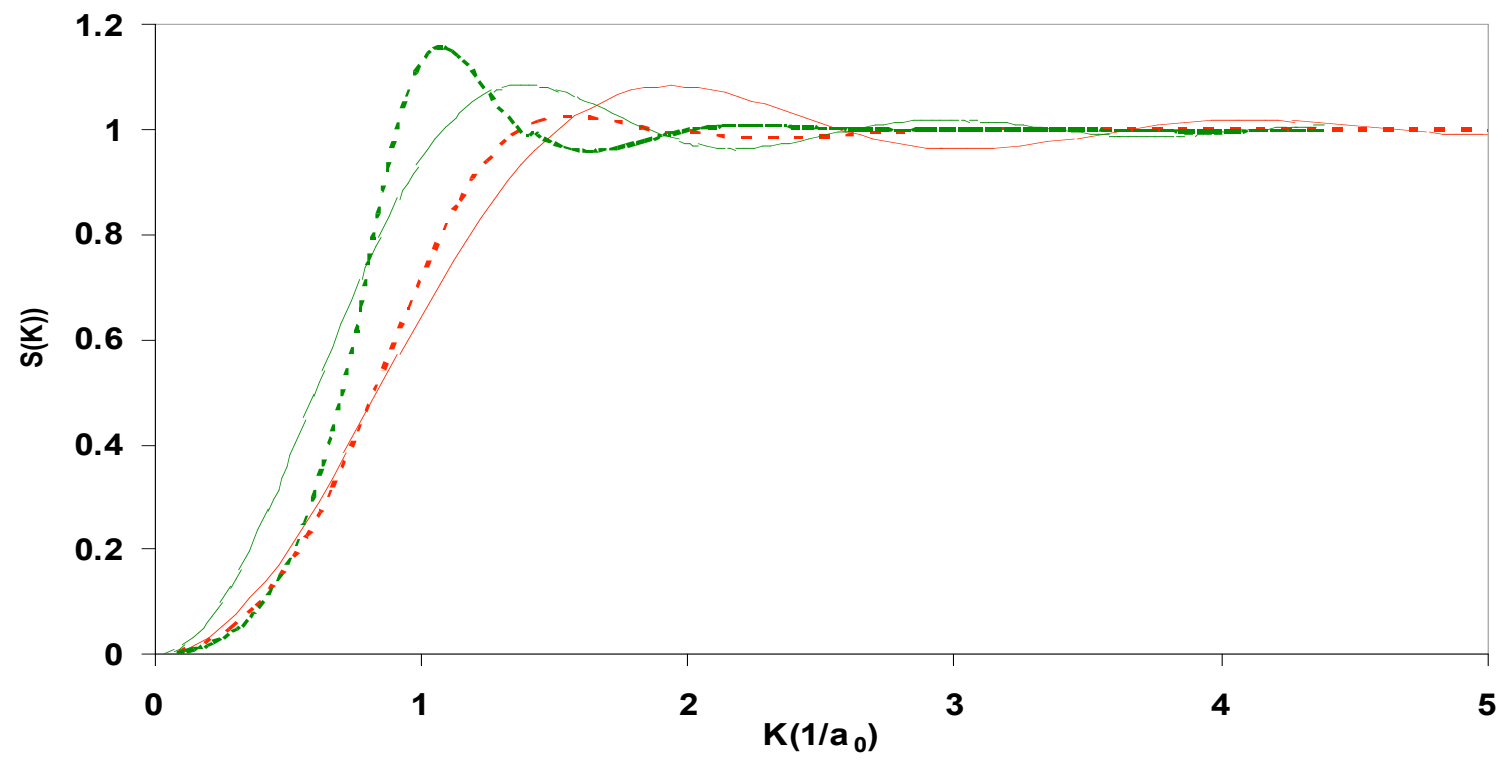

5. Structure factors for two typical cases for aluminum. $\operatorname{Red}: 2.7 \mathrm{~g} / \mathrm{cc}, 10 \mathrm{eV}, \Gamma=4.67$,

Fig. green:1g/cc,0.8617(eV) $(10000 \mathrm{~K}), \Gamma=12.5$. Solid curves - ion-sphere model, dashed curves YCD. 


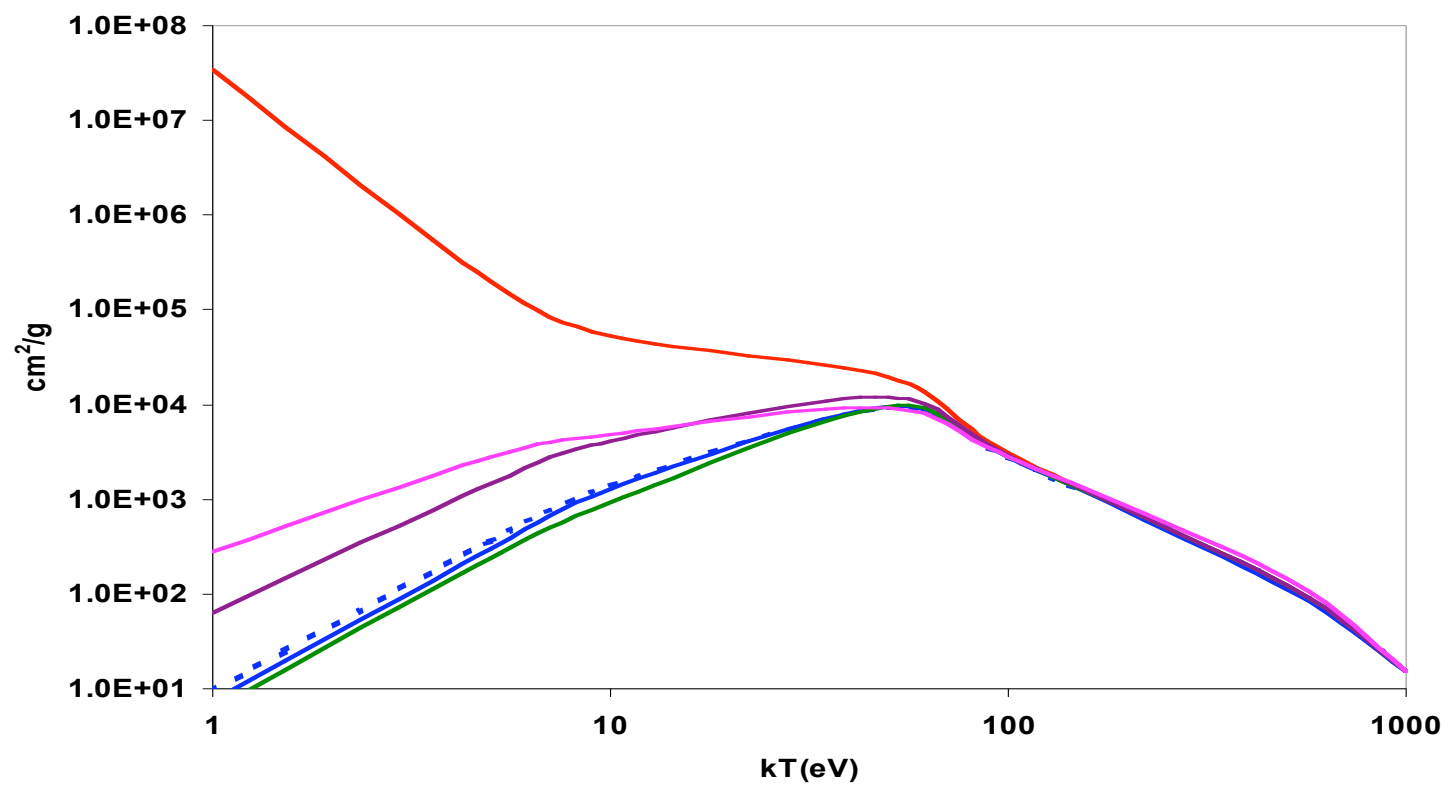

Fig. 6. Effective Rosseland mean opacities versus temperature in aluminum at $2.7 \mathrm{~g} / \mathrm{cc}$ density. Red-radiative only, blue and green, Sommerfeld -Born and quantum mechanical phase shifts, respectively, violet- Born, pink Coulomb scattering. The dashed blue is with $S(K)=1$.
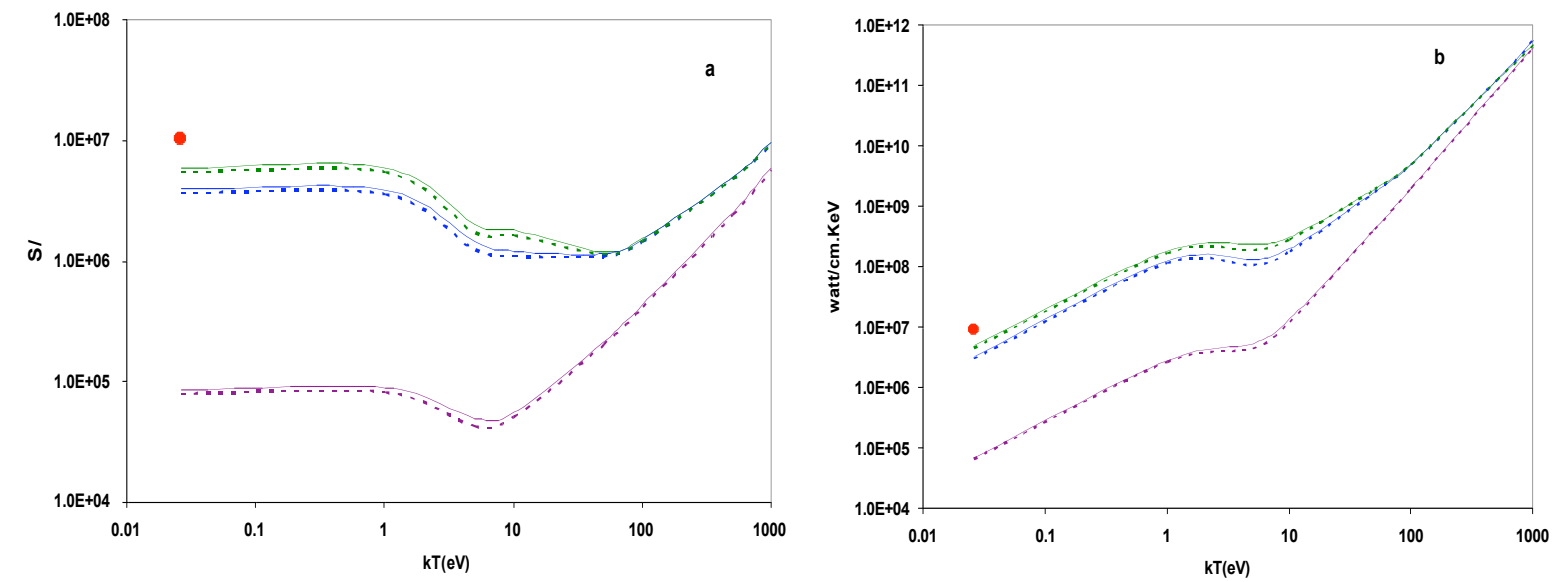

Figure 7. DC electrical (a) and thermal (b) conductivities versus temperature in iron at $7.86 \mathrm{~g} / \mathrm{cc}$ density. The meaning of the colors is the same as in Fig. 3. 


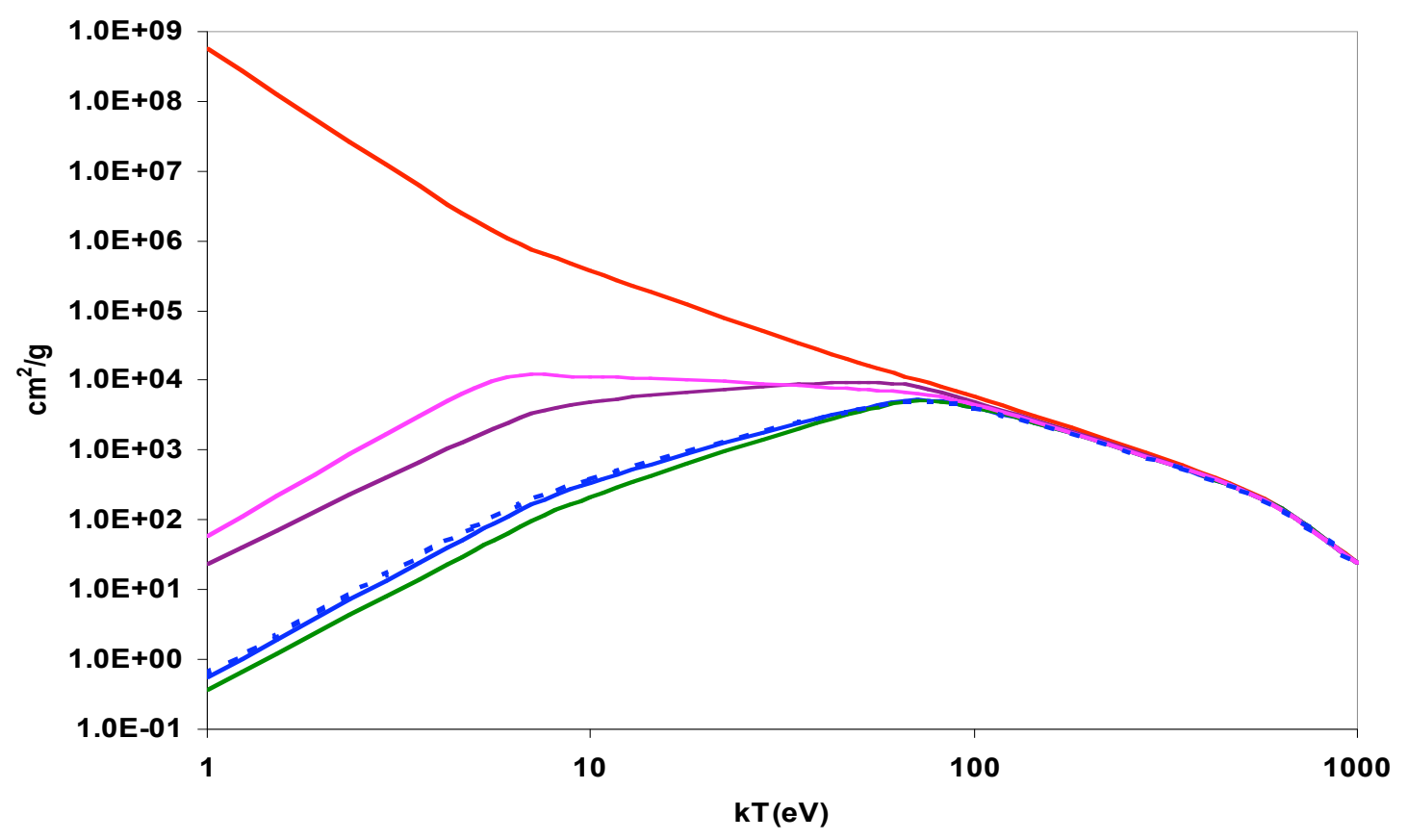

Fig. 8. Effective Rosseland mean opacities versus temperature in iron at $7.86 \mathrm{~g} / \mathrm{cc}$ density. The colors and curves have the same meaning as for aluminum in Fig. 6.
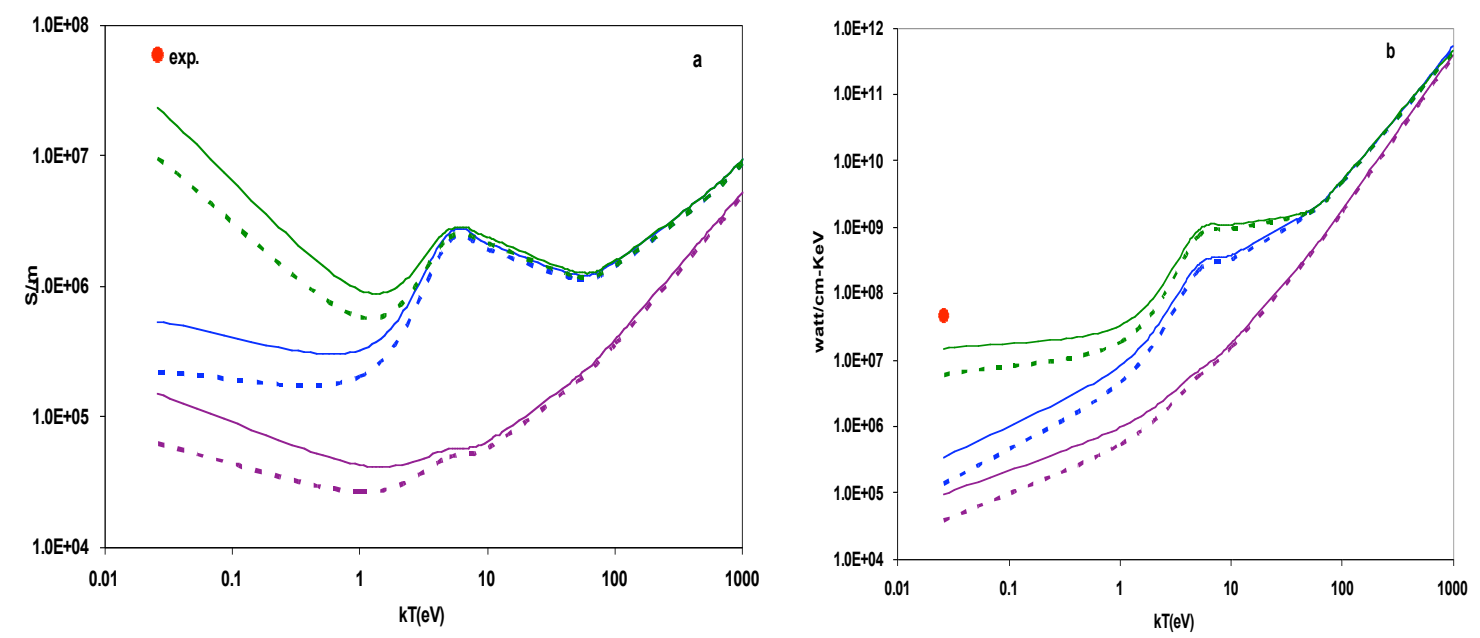

Fig. 9. DC electrical (a) and thermal conductivities versus temperature in copper at 8.96 g/ce density. The colors have the same meaning as in Figs. 3 and 7. 


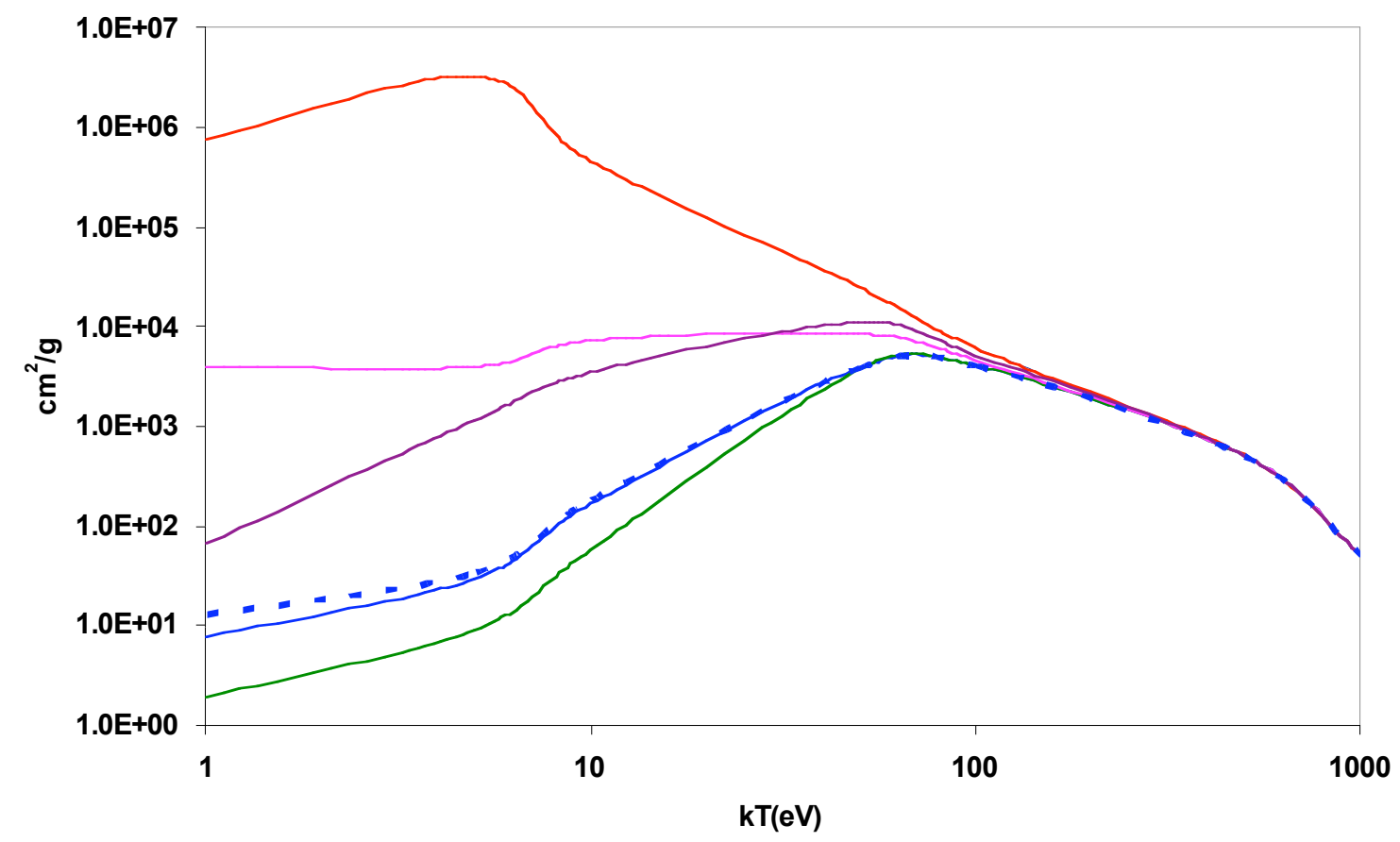

Fig. 10. Effective Rosseland mean opacities versus temperature in copper at $8.96 \mathrm{~g} / \mathrm{cc}$ density. The colors have the same meaning as in Figs. 6 and 8.
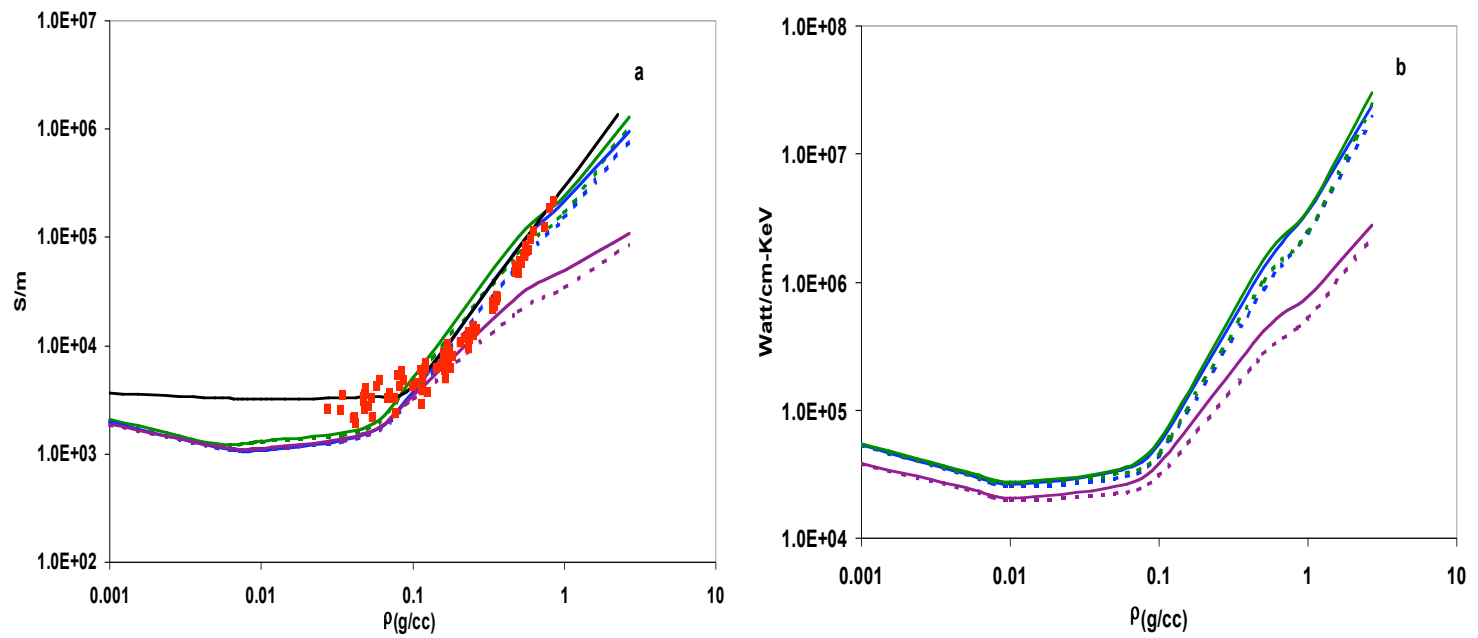

Fig.11. Electrical conductivities in units of $\mathrm{S} / \mathrm{m}$. (a), and thermal conductivities in units of watt/cm-KeV, (b) versus density in aluminum at $10000 \mathrm{~K}$ temperature. The red squares are experimental data and the black curve in (a) is from the Desarlais-Lee-More (DLM) model. Ref.[15]. The rest of 
15

the colors have the same meaning as before.
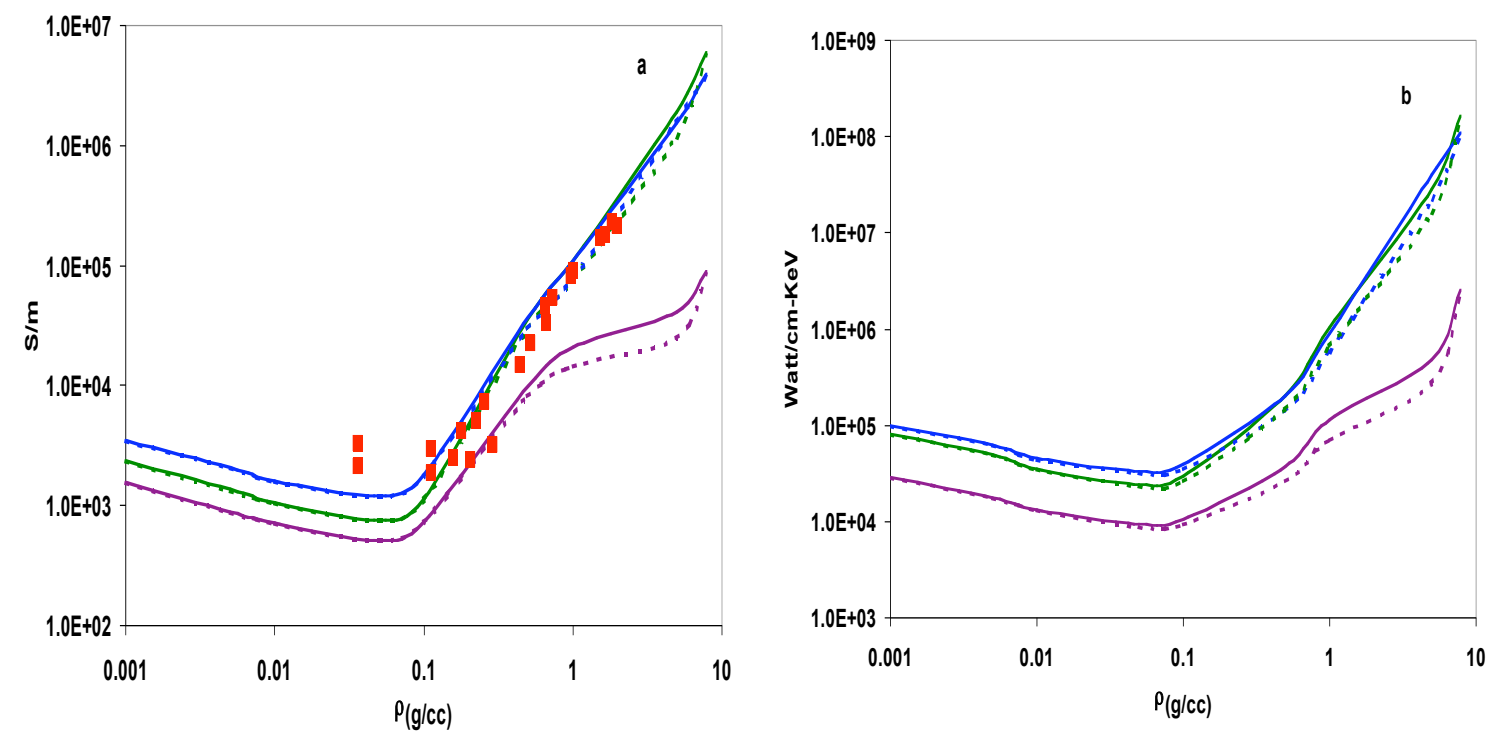

Fig.12 Same as Fig. 11 for iron. Here we do not have the DLM model data.
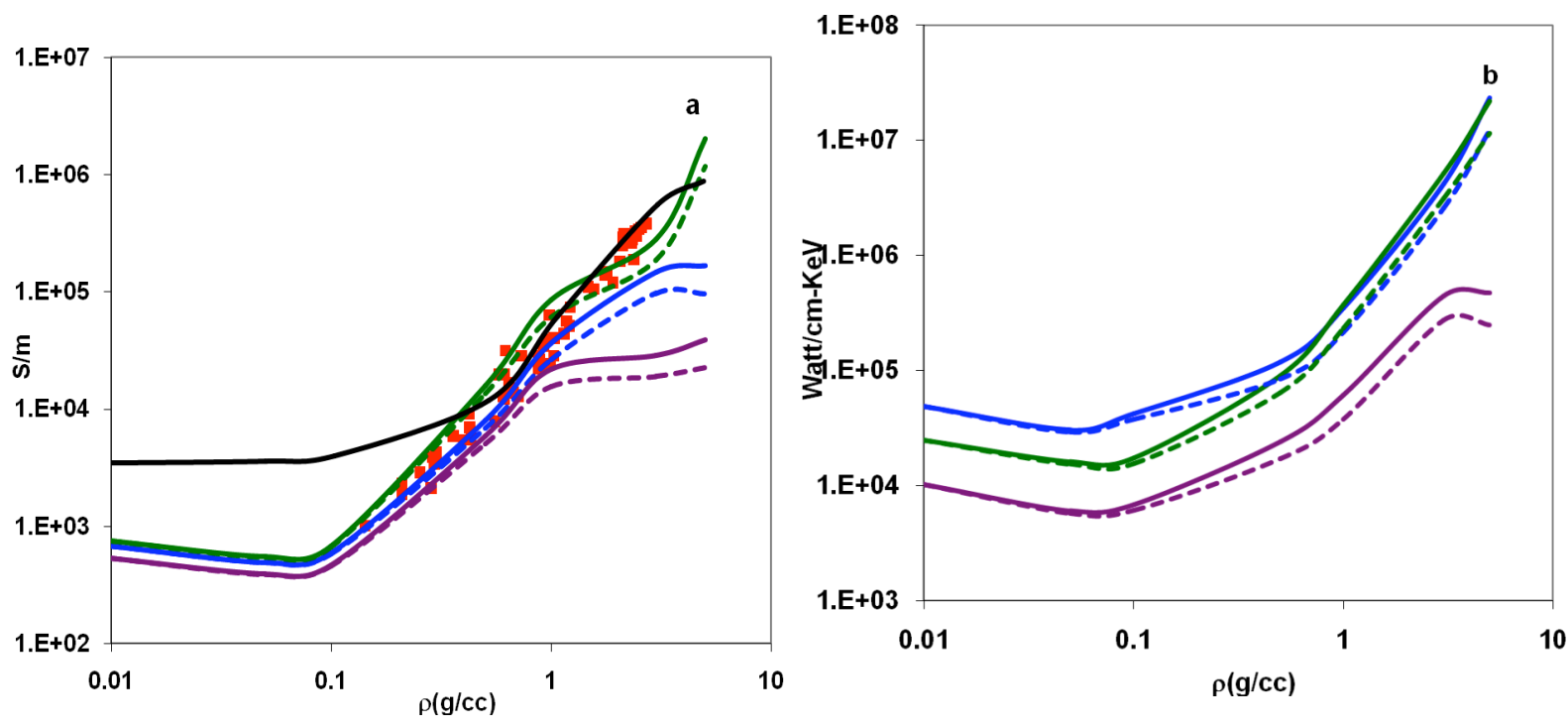

Fig. 13. Same as Fig. 11 for copper. 

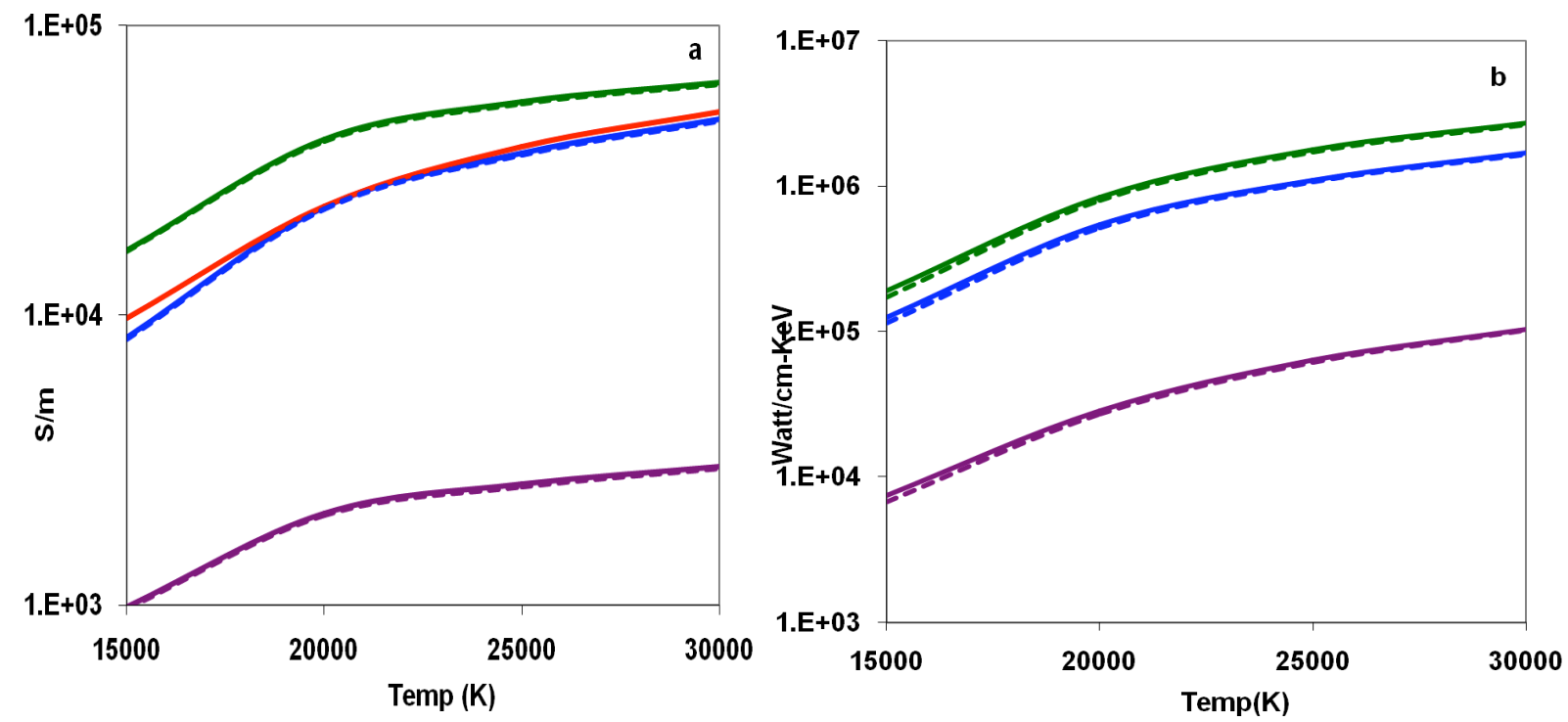

Fig. 14. Electrical (a) and thermal conductivity versus temperature in gold at $0.5 \mathrm{~g} / \mathrm{cc}$. The red curve in (a) is from Ref.[16], the rest of the colors have the same meaning as before. 